\title{
Die reine Spur der Schrift
}

\author{
Unleserliche Schriftzeichen und fingierte Signifikation \\ in Xu Bings Buch des Himmels*
}

\section{Arne KLAWitTer}

\begin{abstract}
$X u$ Bing's imaginary characters haven't stopped irritating their spectators since the first exhibition of his work A book from the sky (1988) in Beijing. For this monumental project, he created about 4000 characters, which, in a certain way, question the status and the tradition of Chinese characters. The characters $X u$ Bing invented are extraordinary because they don't have any meaning: they are empty and without any reference, only pure aesthetical form. At first sight, they look very similar to genuine Chinese characters, but the fact is that they mislead the reader and drive him into a non-signifying void. With Foucault's ontology of language and Derrida's grammatological critique of Western phonocentrism the essay suggests two approaches in order to conceptualize Xu Bing's simulacra of Chinese characters.
\end{abstract}

Title: The >Pure Trace of Writing. Unreadable Characters and Fictive Signification in Xu Bing's Book from the Sky

Keywords: concept of culture; Chinese characters; deconstruction of writing; grammatology; post-structuralism; society; Xu Bing (1955-)

\section{Die Unleserlichen Schriftzeichen IN Xu Bings BÜCHERN DES HimmelS}

Der bis dahin selbst Fachleuten weitestgehend unbekannte chinesische Künstler Xu Bing präsentierte im Oktober 1988 auf einer Kunstausstellung in Peking eine Reihe von Büchern, die mit ihren indigoblauen Einbänden, der weißen Fadenheftung und dem Satzspiegel bis ins Detail die Aufmachung klassischer chinesischer Werken imitierten, ${ }^{1}$ deren Text bei näherem Hinsehen aber von niemandem gelesen werden konnte, denn selbst die scheinbar wohlbekannten

* I Der hier abgedruckte Text ist die überarbeitete Fassung des in Kodikas/Code (35, H. 1-2 [2012]) veröffentlichten Essays Schrift als fingierte Signifikation. Die unleserlichen Schriftzeichen aus Xu Bings "Buch des Himmels".

1 | Zum klassischen Kanon des Konfuzianismus zählen fünf Bücher (die sog. Fünf Klassiker): das Buch der Wandlungen (Yijing), das Buch der Lieder (Shijing), das Buch der Urkunden (Shujing), das Buch der Riten (Liji) und die Frühlings- und Herbstannalen (Chunqiu), eine Chronik der historischen Ereignisse des Staates Lu vom 8. bis zum 5. vorchristlichen Jahrhundert. 
Zeichen erwiesen sich durch zum Teil minimale Veränderungen im Aufbau als unbekannt und damit als unverständlich. Das Kunstprojekt selbst ging unter dem Namen Tianshu, was so viel bedeutet wie »Buch des Himmels« bzw. »Bücher des Himmels «, in die moderne chinesische Kunstgeschichte ein. ${ }^{2}$

Im Westen wurde Xu Bings Werk vor allem als Kritik an der überkommenen chinesischen Schriftkultur und Gelehrtentradition interpretiert. Nicht die Tatsache, dass Xu Bing seine Bücher im Stil kanonischer chinesischer Bücher gestaltet und auf dem Weg der traditionellen Drucktechnik hergestellt hat, ist das Besondere an ihnen, sondern vielmehr die Verbindung des Herkömmlichen mit den dabei verwendeten, ungefähr 1200 Schriftzeichen, die aufgrund ihrer gewollten Verfremdung die Besucher der Ausstellung wohl ausnahmslos in nicht geringes Erstaunen versetzte. Denn obwohl fast jedem von ihnen die Zeichen bekannt schienen, was sogleich zum Versuch animierte, sie zu entziffern, gelang es niemandem, ihnen auch nur irgend einen greifbaren Sinn zu unterlegen. Aufgrund ihrer teilweise frappierenden Ähnlichkeit mit immer noch gebräuchlichen chinesischen Schriftzeichen schienen sie zwar ihnen adäquat zu sein, doch als ein Ganzes waren sie unlesbar. Manche Besucher der Ausstellung fühlten sich getäuscht und hinters Licht geführt, während andere vermuteten, dass es sich hier vielleicht um uralte Schriftzeichen handeln könne, die im Verlauf der historischen Entwicklung und als Konsequenz der letzten umfassenden Schriftreform unter Mao Zedong ihre Lesbarkeit eingebüßt hatten oder zumindest nicht mehr so einfach zu lesen wären. Sollte es demnach dann nicht doch einen Weg geben, wenigstens das eine oder andere dieser Zeichen wiederzuerkennen und es verstehen zu können?

Um es vorwegzunehmen: Nichts dergleichen ist möglich, denn die Schriftzeichen, die Xu Bing in seinen Büchern verwendet, sind allesamt von ihm erfunden. Obwohl sie auf den ersten Blick vielen vertrauten chinesischen Zeichen täuschend ähnlich sehen, unterscheiden sie sich doch in einigen oft nur geringfügigen Details von ihnen, womit sie in letzter Konsequenz überhaupt keinen Sinn mehr ergeben. Xu Bing suchte zu diesem Zweck verschiedene, ihn besonders ansprechende Schriftzeichen aus einem Wörterbuch heraus, ${ }^{3}$ zerlegte sie in ihre Grundbestandteile und setzte diese dann willkürlich neu so zusammen, dass die auf diese Weise zustande gebrachten Gebilde ihre ursprüngliche Bedeutung vollkommen verloren.

Nachdem die Bücher des Himmels 1988 erstmals öffentlich gezeigt worden waren, weitete $\mathrm{Xu}$ Bing sein Projekt in den Folgejahren zu einer Installation

2| Der ursprünglich von Xu Bing gewählte Titel lautete: The Mirror of the World - An Analyzed Reflection on the End of this Century.

3 | So aus dem Shuowen jiezi, das als erstes Zeichenlexikon der chinesischen Sprache um 100 n. Chr. von dem Gelehrten Xu Shen zusammengestellt worden war und das wichtigste Wörterbuch der chinesischen Schrift ist. Es umfasst etwa 10000 Zeichen, die nach 540 Radikalen geordnet sind. 
Abb. 1: Installation Tianshu (>Bücher des Himmels $<$ )

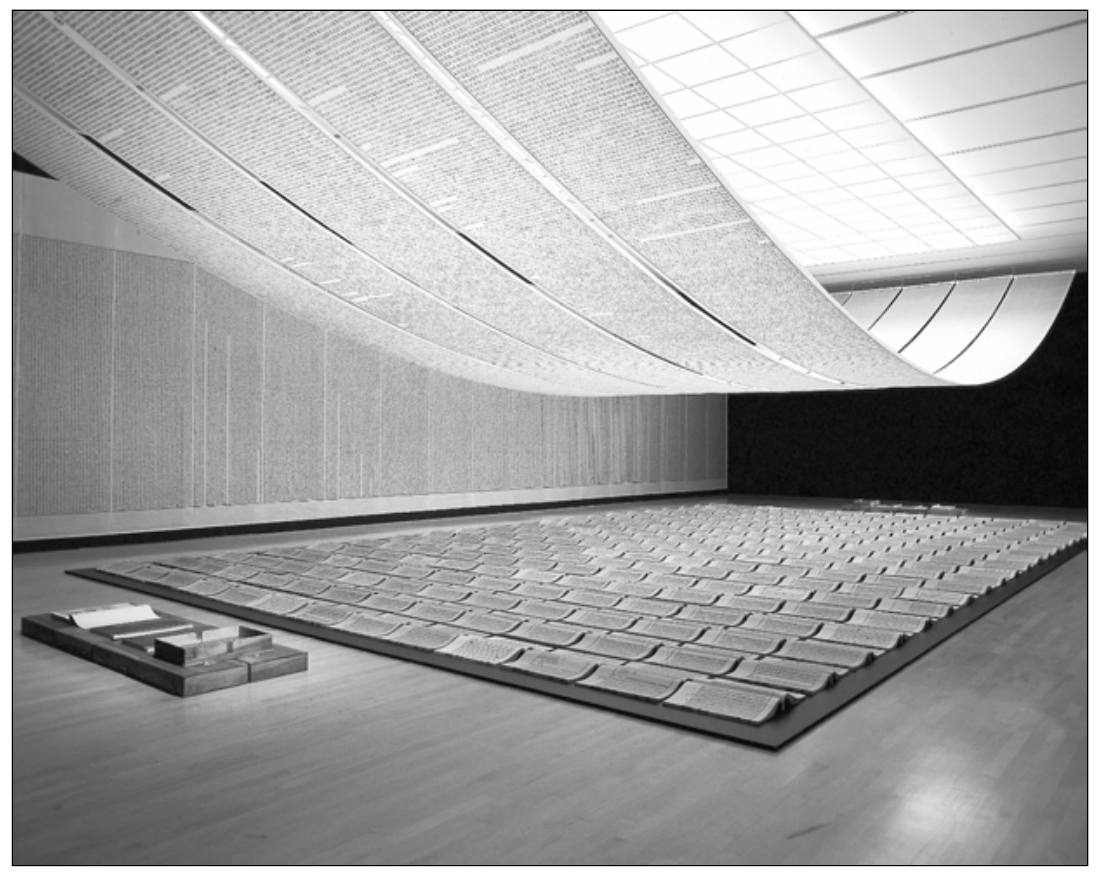

aus, die in den USA, in Japan und anderen Ländern zu sehen war und den Künstler weltweit berühmt machte.

Die Installation bestand aus mehreren riesigen, mit Schriftzeichen bedruckten Papierbahnen, die wie Baldachine von der Decke des Raumes herabhingen und Reminiszenzen an buddhistische Schriftrollen evozierten. Darunter befand sich, ebenerdig ausgelegt, eine auf den ersten Blick unüberschaubare Zahl aufgeschlagener Bücher - ein Arrangement, das durch geschickt eingesetzte Lichteffekte eine faszinierende Atmosphäre erzeugte und mit ihr einen ganz eigenen ästhetischen Eindruck. Mit der schon erwähnten auffälligen weißen Fadenheftung und den indigofarbenen Einbänden folgte $\mathrm{Xu}$ Bing genau den Regeln der traditionellen Buchbinderkunst der späten Song-Dynastie aus dem 11. Jahrhundert. Auch der Druck entsprach exakt dem Vorbild der alten chinesischen Buchdruckerkunst und war mithin ein sehr aufwendiges Unterfangen: Es soll immerhin drei Jahre gedauert haben, bis Xu Bing alle diese neuen Schriftzeichen spiegelverkehrt in jene Holzblöcke geschnitzt hatte, die dem Druck als Vorlage dienten (Abb. 2).

Die Zahl der unlesbaren Zeichen Xu Bings war inzwischen auf gut 4 ००० angewachsen, und das aus einem ganz konkreten Grund: Etwa so viele Zeichen muss ein Chinese lernen und auch beherrschen, um seine tägliche Alltagslektüre bewältigen zu können.

Besonders wichtig für eine zeichentheoretische Betrachtung dieser neu geschaffenen Schriftzeichen scheint mir der Gesichtspunkt zu sein, dass sie bewusst dahingehend manipuliert worden sind, keine wirklichen Zeichen mehr 
Abb. 2: Druckstock des Frontispizes aus Tianshu

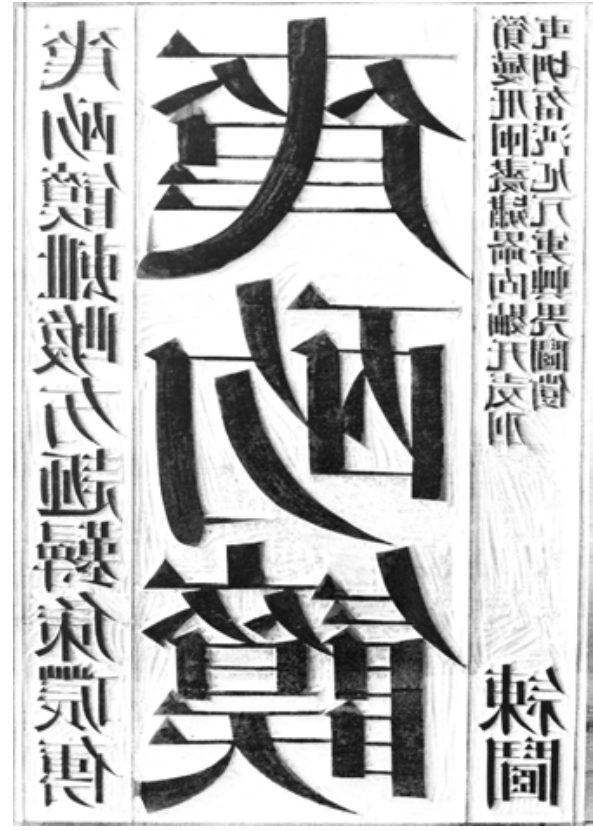

Abb. 3: Buchseite aus Tianshu

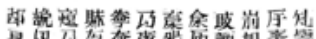

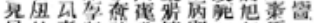

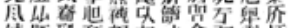

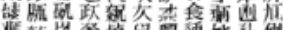

\begin{tabular}{|c|c|c|c|c|}
\hline 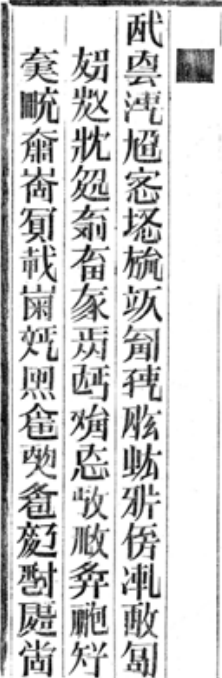 & 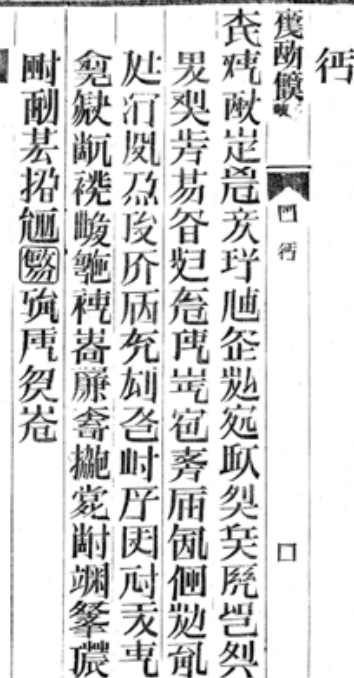 & 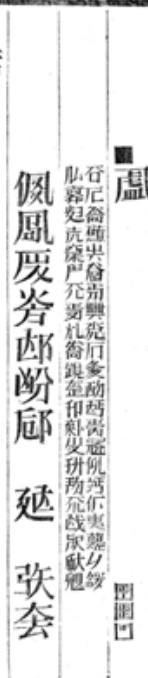 & 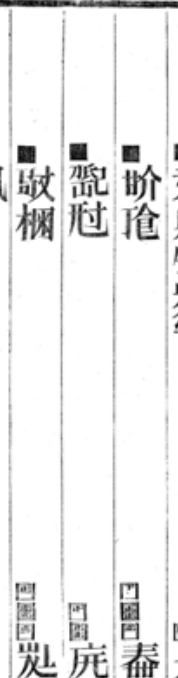 & 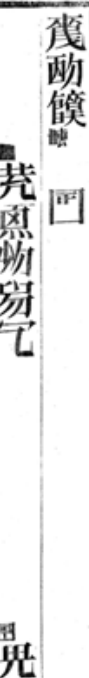 \\
\hline
\end{tabular}


zu sein. Vom optischen Eindruck her scheinen sie zwar zu signalisieren, dass es sich durchaus um Zeichen mit einer konkreten Sinngebung handeln könnte, doch referieren sie auf nichts: Sie haben weder einen semantischen Wert, noch können sie mit einem Laut in Verbindung gebracht werden, und dies, obwohl doch jede ihrer einzelnen Komponenten ganz und gar konventionellen Zeichenelementen entspricht. Da aber die prinzipielle Zeichenhaftigkeit dieser eigentlich unlesbaren Zeichen sehr wohl erkennbar ist, spreche ich hier in diesem Zusammenhang von >unleserlichen Zeichen<.

Den folgenden Betrachtungen liegen zwei verschiedene Zeichenkonzeptionen zugrunde, die in Abgrenzung zum strukturalistischen Zeichenbegriff zweierlei Optionen anbieten, um den Zeichencharakter unter dem Aspekt der Nicht-Signifikation zu problematisieren: Michel Foucaults Sprachontologie und Jacques Derridas Grammatologie.

\section{Foucaults sprachontologische Perspektive}

Die grundsätzliche Frage, die Xu Bing mit seinen abgewandelten Schriftzeichen aufwirft, richtet sich auf die Schwelle zwischen einer bloßen Strichfolge und einem signifizierenden Zeichen. Was dergleichen Schriftzeichen aus semiotischer Perspektive so interessant macht, ist, dass sie die Grenze zwischen der Signifikation und dem Nichtsignifikativen erkunden, womit sie eine Reihe von Fragen hervorrufen: Unter welchen Bedingungen ist ein Zeichen ein Zeichen? Welche Funktion haben unleserliche (was auch bedeuten kann: archaische) Zeichen in der Zeichenwelt einer Gegenwartssprache? Inwieweit können archaische Zeichen noch verstanden werden und unlesbare Zeichen lesbar gemacht werden, und welche Konsequenzen ergeben sich daraus für den Referenzzusammenhang? Was geschieht mit Schriftzeichen, wenn man sie modifiziert, und welcher Spielraum ist dafür erlaubt? Inwiefern sind sie dann überhaupt noch Zeichen, und sind sie auch dann noch Zeichenträger (Signifikanten), wenn das Signifikat nicht mehr unmittelbar präsent ist? Von welcher Art sind die Signifikanten, die ihr Signifikat eingebüßt haben? Welche Rolle spielen sie in der Zeichenwelt unserer Gegenwartssprache? Inwiefern können solche an sich schon zweifelhaften Signifikanten eine andere Bedeutung annehmen? Und inwiefern sind sie konstitutiv für das, was wir im Zeitalter der Moderne als Kunst bzw. Literatur verstehen?

Auf all dies eine befriedigende Antwort zu geben, ist hier natürlich nicht möglich, doch soll im Folgenden zumindest auf diejenigen Fragen näher eingegangen werden, die sich ergeben, wenn man Xu Bings modifizierte Zeichen mit Foucault als ontologische Hinweise auf das nicht-signifikative Sein der Sprache begreift.

Im Vorfeld seiner Diskursanalyse und Wissensarchäologie arbeitete Foucault in einer Reihe von Essays und Rezensionen in den Jahren zwischen 1961 und 1966 eine ganz eigene Literaturontologie aus, der in der Forschung jedoch wenig Beachtung geschenkt worden ist. Er stützte sich dabei auf Überlegun- 
gen von Maurice Blanchot, der in der modernen Literatur (seit Baudelaire und Mallarmé) eine »machtvolle Negationsbewegung « am Werk sah und eine Diskursweise, die sich »über ihren Trümmern errichtet« (Blanchot 1993: 12). Seiner Meinung nach schöpft die moderne Literatur gerade aus ihrer Nichtigkeit (d.h. der >Abwesenheit des Werkes < und dem Entzug von Sinn) eine »wunderbare Kraft«, durch die sie vermag, darauf hinzuwirken, »daß sie sich rückhaltlos jenem Teil öffnet, den das Nichts an ihr hat, daß sie ihre eigene Unwirklichkeit verwirklicht« (ebd.). Darauf aufbauend, formulierte Foucault den Gedanken, dass in der modernen Zeit die Literatur das sei, »was das signifikative Funktionieren der Sprache kompensiert (und nicht bestärkt)« (Foucault 1971: 77). Die Radikalität dieser These ist häufig übersehen worden. Foucault weist hier nämlich dem nicht-signifikativen Moment eine konstitutive Rolle für die moderne Literatur zu. Gleichzeitig erhält diese eine besondere Funktion in Bezug auf die epochale Wissensformation (Episteme), die ohne die in Foucaults früheren Schriften zur Literatur dargelegten literaturontologischen Prämissen kaum zu verstehen ist. Sie besagen, dass seit dem 19. Jahrhundert - von Hölderlin über Mallarmé bis zu Artaud und Blanchot - die Literatur als eine Art »Gegendiskurs« funktioniere, indem sie »von der repräsentativen oder bedeutenden Funktion der Sprache zu jenem rohen Sein zurückging« (ebd.: 76).

In seinem Aufsatz Die Sprache, unendlich umreißt Foucault sein Konzept moderner Literatur, mit dem, ausgehend von den »großen ontologischen Ereignissen der Sprache « (Foucault 2003: 87), ein Raum sichtbar gemacht wird, in dem Sprache und Tod sich kreuzen und die Sprache ihre Endlichkeit reflektiert. Dabei bezieht sich Foucault vor allem auf den achten Gesang von Homers Odyssee, auf die Rahmenhandlung der Märchen aus Tausendundeiner Nacht sowie auf die Erzählung Das geheime Wunder von Jorge Luis Borges und verdeutlicht, wie an der Grenzlinie des Todes die Rede auf eine Art Sprachspiegel trifft, und, indem sie versucht, den Tod aufzuhalten, in diesem Spiegel ihr eigenes Bild entstehen lässt. In der Tiefe des Spiegels, wo sich die Rede verliert und sie ihre Signifikation einbüßt, wird man nach Ansicht Foucaults eines anderen Sprechens gewahr, und zwar als »winziges, inneres und virtuelles Modell«, das eine enge Verknüpfung von Tod, grenzenlosem Sich-selbst-Verfolgen und Selbstdarstellung der Sprache impliziert (ebd.: 87).

Vor dem Hintergrund der Feststellung, dass die Verdoppelung der Sprache, selbst wenn sie im Werk noch verborgen ist und nur durch Risse an die Oberfläche dringt, konstitutiv ist für das sprachliche Werk und erst recht für die Literatur, wo sich die Verdoppelung zu einem unendlichen Sprechen ausweitet, folgert Foucault, dass die Zeichen, die auf diese Weise zutage treten, »als ontologische Hinweise « (ebd.: 89) zu lesen seien. Das besagt nichts anderes, als dass die Figuren der Sprachverdoppelung, wie sie beispielsweise als Spiegelungen oder Verschachtelungen innerhalb eines Werkes auftreten können, nicht als Momente der Selbstreferenz aufzufassen sind, sondern im Sinne einer Selbstimplikation des Sprechens. Foucault führt diesen Begriff bewusst im Gegensatz zum strukturalistischen Konzept der Selbstreferenzialität ein, das Zeichen voraussetzt, die auf ihr Funktionieren in einem Zeichenzusammenhang verwei- 
sen und damit zeigen, dass sie zeigen. Im Gegensatz zur Selbstreferenzialität, die ein Sprechen über einen Code impliziert, handelt es sich beim selbstimpliziten Sprechen gewissermaßen um ein Sprechen ohne Code: um einen Diskurs, der keine Entzifferung verlangt, weil er nichts kodiert. In diesem Fall wird der Verweisungszusammenhang suspendiert; es handelt sich demnach bei den >Zeichen $<$, die in Erscheinung treten, genaugenommen nicht mehr um Signifikanten, denn ihre Funktion besteht, wie schon oben angedeutet, nunmehr darin, $\mathrm{zu}$ zeigen, dass sie nicht zeigen. Foucault zieht daraus den Schluss, dass diese Figurationen der Sprachverdoppelung, diese unendlichen Spiegelungen und Redeformen des Unendlichen, als Indikatoren eines nicht-signifikativen Seins der Sprache aufgefasst werden können, was sich als eine Aufforderung verstehen lässt, sie als ontologische Hinweise zu lesen.

\section{Fingierte Signifikation}

Mit Zeichen, die das Sein der Sprache indizieren, hat man es dann zu tun, wenn diese >Zeichen < signalisieren, dass sie Zeichenformen sind, ohne aber tatsächlich auf etwas Außersprachliches zu referieren bzw. einen Sinnzusammenhang zu vermitteln. Das wäre z.B. bei einer unleserlichen Handschrift der Fall, die die Vermutung nahe legt, dass sie sehr wohl etwas bedeutet, doch mit der Einschränkung verbunden ist, dass sie sich nicht entziffern lässt. ${ }^{4}$ Eine andere Situation tritt jedoch dann ein, wenn die Zeichen bewusst so arrangiert oder manipuliert werden, dass sie eine Signifikationsfunktion fingieren. Genau das trifft auf die konstruierten Schriftzeichen von Xu Bing zu.

Abb. 4: Beispiel eines fingierten Schriftzeichens

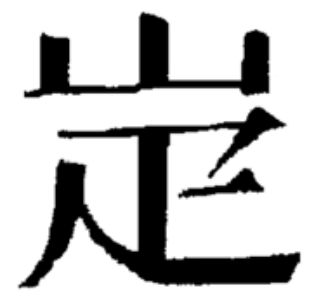

4 | Erwähnen könnte man hier Robert Walsers Mikrogramme, die in einer Miniaturschrift geschrieben sind, von der man zunächst annahm, dass sie eine "nicht entzifferbare Geheimschrift" (Carl Seelig, zit. n. Middleton 1957: 46) sei. Tatsächlich handelt es sich jedoch um eine ins Extreme verkleinerte Sütterlinschrift, die seit den 1970er Jahren nach und nach durch Jochen Greven und Martin Jürgens entschlüsselt und transkribiert wurde, so dass die Texte (darunter einige bis dahin unbekannte) der Forschung zugänglich gemacht werden konnten. In den 1980er Jahren begannen dann Bernhard Echte und Werner Morlang mit der systematischen Transkription des gesamten Mikrogramm-Konvoluts, das 526 Blätter umfasst (vgl. Walser 2003). 
Ein solches konstruiertes >Kunst-Zeichen< ist erwartungsgemäß in keinem der einschlägigen Wörterbücher zu finden, die man befragen könnte. Es hat weder Bedeutung noch Lautung, obwohl es aus zwei Komponenten zusammengesetzt ist, die es, jede für sich genommen, im Chinesischen tatsächlich gibt: Im oberen Teil steht unübersehbar das Zeichen für >Berg<山 (»shan«) und darunter das Zeichen für >Stoffballen< oder >eine Rolle aus Stoff<正 (»pi«). Auch wenn seine beiden Komponenten als Schriftzeichen jedes für sich sehr wohl existieren, gibt es das Zeichen als Gesamtheit nicht. Der Betrachter könnte hier zwar eine Bedeutung imaginieren, wie z.B. >ein Berg von Stoffballen<, aber diese Bedeutung wäre rein subjektiv und in keiner Weise lexikalisch abgesichert. Entscheidend aus sprachontologischer Sicht ist, dass durch die konventionellen Elemente dieses fingierten Zeichens suggeriert wird, es als sprachliches Zeichen aufzufassen. Doch da es dieses Zeichen als Ganzes realiter so gar nicht gibt, besteht seine Funktion allein darin, sein sprachliches Sein (sowie das mögliche Bedeutungspotential dieses Sprache-Seins) zu indizieren.

Für Foucault sind die Spiegelungen und Verdopplungen, die er in literarischen Texten ausfindig macht, Figurationen, die eine semantische Entleerung bewirken und die Sprache in ihrem leeren, d.h. nicht-signifikativen Sein zur Darstellung bringen. In solchen Fällen hat man es also strenggenommen nicht mehr mit Signifikanten zu tun, die mit einem Signifikat verbunden sind. Im Poststrukturalismus bezeichnet man diesen Sachverhalt als Trennung zwischen Signifikant und Signifikat: »Wenn der Strukturalismus das Zeichen von seinem Referenten getrennt hat, so geht diese Denkweise - oft unter dem Namen >Poststrukturalismus < bekannt - noch einen Schritt weiter: sie trennt den Signifikanten vom Signifikat.« (Eagleton 1988: 111) Foucault hat mit den Poststrukturalisten wie Derrida und dem späten Barthes gemein, dass seine speziell auf die moderne Literatur zugeschnittene Sprachontologie das linguistische Zeichenmodell von Saussure sprengt. Während aber für die Textualisten die Bedeutung entlang der Signifikantenkette verstreut ist, sieht Foucault sie im Spiel der Verdopplungen in einer Sinn-Reserve zurückgehalten - Reserve aber nicht als Vorrat verstanden, sondern als Figur eines Rückhalts, »die den Sinn zurückund in der Schwebe hält und eine Leere einrichtet, in der allein die noch nicht vollzogene Möglichkeit so zur Vorlage kommt, dass irgendein Sinn sich darin niederlässt, oder irgendein anderer, oder gar noch ein dritter, und dies vielleicht in unendlicher Folge $\ll$ (Foucault 2003: 182).

Was Foucault im diskursiven Zusammenhang anhand der modernen Literatur erläutert und verdeutlicht, trifft auf die Zeichenmodifikationen von Xu Bing im besonderen Maße zu. Seine Konstrukte verweisen weder auf ein Korrelat, d.h. auf ein Signifikat, noch auf den Zeichenzusammenhang, in dem sie funktionieren. Sie laden den Betrachter aufgrund ihres konventionellen Aussehens zunächst zu einer Entzifferung ein, machen die Entzifferung jedoch im gleichen Augenblick unmöglich. Niemand kann ihnen eine definitive Bedeutung zuweisen oder vermag es, ihnen einen Sinn zu entlocken. Was aber noch wichtiger ist: Sie sind unaussprechbar. Mit Buchstaben geschriebene Texte können wir, sofern wir das Alphabet kennen, nicht nur problemlos lesen, sondern auch 
aussprechen, selbst ohne den Sinn der Wörter zu verstehen. Das ist bei einem weitgehend logografischen Schriftsystem wie dem chinesischen nicht der Fall.

In seinem Aufsatz Die Sprache, unendlich hat Foucault angemerkt, dass die alphabetische Schrift in sich bereits eine Form von Verdopplung sei, »da sie nicht das Signifikat repräsentiert, sondern die phonetischen Elemente, die es bedeuten«, während das Ideogramm direkt das Signifikat vorstelle, »unabhängig vom phonetischen System, das eine andere Art der Repräsentation ist « (ebd.: 88). Linguisten werden dem vermutlich entgegenhalten, dass die chinesische Schrift ebenso wie die der westlichen Sprachen sehr wohl phonetische Elemente repräsentiere. Dem aber steht ein gewichtiges Argument entgegen, nämlich jenes, das belegt, dass ein und dieselbe chinesische Schrift für ganz unterschiedliche phonetische Systeme benutzt wird, d.h. sowohl für das Hochchinesische (Mandarin) als auch für das Kantonesische. Hinzu kommt, dass man, wenn man Chinesisch gelernt hat, eine Vielzahl sino-japanischer Schriftzeichen (Kanji) lesen und verstehen kann, ohne zu wissen, wie sie ausgesprochen werden. Dabei resultiert die Bedeutung direkt aus dem Schriftzeichen selbst ohne irgend einen >Umweg über ein phonetisches System. Für die sprachontologische Betrachtung sowohl wirklicher chinesischer Zeichen als auch der konstruierten Zeichen $\mathrm{Xu}$ Bings ergeben sich daraus wichtige Konsequenzen.

Gegen das ideogrammatische Argument ließe sich wiederum einwenden, dass die chinesischen Schriftzeichen mehrheitlich eben keine Ideogramme seien, sondern Phonogramme. Dabei handelt es sich vor allem um mehrteilige Zusammensetzungen, die ein Radikal besitzen, das als sogenanntes >Wurzelzeichen< die Bedeutung des Zeichens bestimmt (deshalb oft auch als >Signifikum < bezeichnet), und ein Phonetikum, d.h. dessen lauttragenden Teil. Das Phonetikum findet sich häufig auf der rechten Seite des Schriftzeichens, ohne dass dies jedoch die Regel wäre: Es kann gegebenenfalls auch links, oben oder unten stehen, woraus sich ergibt, dass ein Zeichen nur lesbar ist, wenn man es schon kennt. Obwohl circa $90 \%$ der Schriftzeichen ein Phonetikum enthalten, gehen Sprachwissenschaftler davon aus, dass ein Leser eine nicht mehr als maximal vierzigprozentige Chance hat, die Aussprache eines ihm unbekannten Schriftzeichens zu erraten. Für ihn gibt es also kaum eine andere Möglichkeit, als alle Schriftzeichen auswendig zu lernen (vgl. Taylor/Taylor 1983: 40).

Dies alles trifft aber auf die von Xu Bing aufs Neue aus altbekannten Komponenten zusammengesetzten Schriftzeichen nicht zu, denn sie beziehen sich, wie wir gesehen haben, ja keineswegs auf eine gesprochene Sprache, sondern breiten sich auf den Buchseiten aus, indem sie unentwegt die grafischen Formen bekannter und überkommener Zeichen abwandeln. Gerade der Umstand, dass die einzelnen Elemente seiner konstruierten Zeichengebilde durchaus lesbar sind, diese als Ganzes aber nicht ausgesprochen werden können, lenkt die Aufmerksamkeit auf ihren ideogrammatischen Gehalt. In den Büchern des Himmels gibt es dann allerdings keine Zeichen im eigentlichen Sinne mehr, sondern nur noch deren stumme Rohformen: die genuine Möglichkeit von Zeichen. Man könnte sie als Krypto-Zeichen beschreiben oder als Zeichensimulakren, d.h. als Trugbilder von Zeichen, weil mit ihnen die Illusion geschaffen wird, dass ih- 
nen eine Bedeutung innewohnen könne, ja, womöglich sogar innewohne - nur, dass wir nicht in der Lage sind, diese zu erkennen. Xu Bings Zeichengebilde als ontologische Hinweise aufzufassen, hieße also, sie als Zeichenfigurationen zu verstehen, die dem Betrachter signalisieren, dass sie nicht zeigen, und die am Nullpunkt ihrer Signifikation einzig das Sein der Sprache indizieren.

\section{Xu Bings Zeichenexperimente und ihre Verfahren}

Xu Bings konstruierte Schriftzeichen sind keineswegs die einzigen Zeichenfigurationen in der chinesischen Kulturgeschichte, durch die Sprache in ihrem opaken Sein in bildliche Darstellung überführt wird. Wie der Sinologe Robert E. Harrist gezeigt hat, gibt es in der ostasiatischen Kunstgeschichte schon viel früher Formen von Virtual writing, wie z.B. in den im 13. Jahrhundert entstandenen Schriftrollen des Bilderzyklus' Die zehn Könige der Hölle. Dort sieht man auf dem siebten Bild einen König, der gerade dabei ist, solche Rollen zu überprüfen, um über das Schicksal der ihm zugeteilten Seelen zu urteilen. Diese ihm von Dämonen überreichten Schriftrollen sind angefüllt mit unleserlichem Gekritzel. Der erste optische Eindruck lässt an eine flüchtige Kursivschrift denken, aber tatsächlich ist es eine auf keine Weise lesbare Schrift. Harrist hat außerdem darauf hingewiesen, dass, wenn Texte in ein Bild integriert sind, sie in der Regel Hinweise für die Interpretation des Bildes geben. In diesem Falle aber liege der Verwendung einer solchen >virtuellen Schrift< ein ganz anderes Konzept zugrunde: »[...] while the screens and books in these paintings are representations of real objects, the writing is real writing, which suggests that the only way to make a representation of writing that is not real writing is to adopt the method of the Ten Kings of Hell painting and replace legible texts with illegible lines« (Harrist 2006: 35f.).

$\mathrm{Xu}$ Bing kommt es jedoch auf etwas anderes an: Indem er nicht die Schrift virtualisiert, um auf den Charakter der Repräsentation zu verweisen, sondern die Signifikation der Zeichen derart minimiert, dass lediglich ausgeleerte, stumme Zeichenmonumente übrig bleiben, greift er weit über ein reines Spiel mit Zeichen oder ein semiotisches Gedankenexperiment hinaus. Die nicht-signifikativen Zeichengebilde sind für Xu Bing eine Art Versuchsanordnung, mit deren Hilfe er die Welt der Zeichen untersucht, wie es ja auch der ursprüngliche Titel der Installation nahelegt. Es geht dabei also nicht allein um die Selbstrepräsentation von Schrift, sondern vielmehr um die Infragestellung einer Schrift- und Kulturgeschichte, denn dadurch, dass er die einem jeden Chinesen bekannten grafischen Zeichenelemente in unlesbare Zeichensimulakren verwandelt, verfremdet er sie und trennt sie abrupt von der langen Kulturgeschichte. Darüber hinaus macht er jegliche Kommunikation unmöglich, indem er die Betrachter seines Kunstwerkes im buchstäblichen Sinne sprachlos werden lässt.

Harrist hat darauf hingewiesen, dass Xu Bing bei der Schaffung seiner fingierten Zeichen genau jene Regeln beachtet, denen Psychologen folgen, wenn 
sie Pseudozeichen für ihre psychologischen Experimente kreieren, indem er nämlich wie diese ganz besonders darauf achtet, dass die Radikale in den ihnen zugewiesenen Positionen verbleiben, wie man es von den gewohnten Zeichen her kennt. ${ }^{5}$ Das Zeichen 江 (»jiang«, dt. >Fluss $<$ ) mit dem Radikal >Wasser < wird für ein psychologisches Experiment dahingehend verändert, dass der linke Teil, der für >Wasser $<$ steht, mit dem Zeichen (Radikal) für >Jade<玉 (»yu«) verknüpft wird, wobei das Zeichensimulakrum シ 玉 entsteht. Daraus könnte man einen Begriff wie etwa >Wasserjade< herauslesen, aber das Zeichen, so wie es hier geschrieben steht, gibt es nicht: Es ist ein völliges Novum. Ein weiteres Beispiel wäre das Zeichen 姐 (»jie«), das >ältere Schwester < bedeutet. Während der linke Teil mit der Bedeutung >Frau< erhalten bleibt, wird beim Experiment der rechte Teil des Zeichens durch 足 ersetzt. Es handelt sich dabei um einen Bestandteil des Zeichens für >Zehe<: 趾 (»zhi«). Aber auch dieses Zeichen gibt es in dieser Schreibweise 女足 nicht. Man könnte bei seiner Ausdeutung zudem einer Assoziation folgen, die sich aus der horizontalen Anordnung der Radikale auf der rechten Seite ergibt: 口止. Das Zeichen 口 (»kou«) hat die Bedeutung >Mund $<$, und 止 (»zhi«), zugleich die lauttragende Komponente des Zeichens, bedeutet so viel wie >anhalten $<$, >stoppen $<$, >verbieten $<$, >verhindern $<$. 口止 könnte demnach so viel wie >nicht sprechen < oder >aufhören zu sprechen< heißen und die Bedeutung >eine Frau, die zu sprechen aufhört<, assoziieren.

Ein ähnliches Verfahren hat auch Xu Bing verwendet, was sich an dem oben bereits erwähnten Beispiel aus den Büchern des Himmels zeigen lässt. Unterlegt man dem fingierten Zeichen (Abb. 4) eine solche Intention, dann wäre als Ausgangszeichen 岩 (»yan«) anzunehmen, das sich aus den Komponenten 山石 (>Berg< und >Stein<) zusammensetzt und so viel wie >Felsen< bedeutet. Das obere Zeichen für >Berg< bliebe hierbei erhalten, während das untere von $\mathrm{Xu}$ Bing ausgetauscht wird, und zwar durch das Zeichen 疋 (»pi«), das so viel bedeutet wie >Stoffballen< oder >eine Rolle aus Stoff<.

Abb. 5: Fingierte Schriftzeichen auf einer Seite des Tianshu

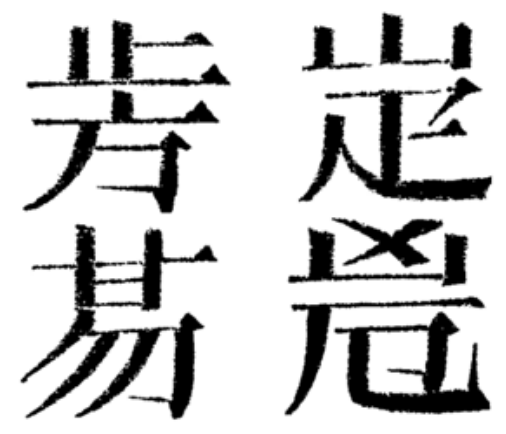

Das Verfahren ließe sich auch auf andere Zeichenmodifikationen anwenden. Die Neuschöpfung oben links in der Abbildung 5 setzt sich aus zwei Teilen

5 | Vgl. den Aufsatz von Weekes (1997: 171-186), auf den sich Harrist beruft. 
zusammen: Der obere Teil besteht aus dem Zeichen 止 (»zhi«), was so viel wie >aufhören < bedeutet, und der untere Teil aus dem Zeichen 方 (»fang«) in der Bedeutung von >Richtung $<$ oder auch >Quadrat<. Beide sind zwar reale Zeichen, doch gibt es im Chinesischen kein in dieser Kombination zusammengesetztes Zeichen. Das Gleiche gilt für das darunter stehende Zeichengebilde, das ebenfalls aus zwei Teilen besteht, in dem das obere Zeichen 甘 (»gan«) >süß <edeutet und das untere Zeichen 勿 (»wu«) so viel wie >nicht< oder >nein<. Man könnte das Ganze dahingehend interpretieren, dass man nichts Süßes essen solle, doch auch für diese Zeichenkombination gibt es keinerlei Vorbild in der Realität.

Das vierte Zeichen unten rechts schließlich scheint auf den ersten Blick eine Modifikation des realen Zeichens 危 (»wei«) zu sein, das so viel wie >Gefahr<, >Unheil< oder >Unglück < bedeutet und hier gleichsam verstärkt wird durch das Zeichen \ (»xiong«) mit einer ähnlichen Bedeutung. Letzteres leitet sich vom Radikal 山 (»qu«) für >Grube<, >Behälter < oder >geöffneter Mund $a b$ und bezeichnet eine »unachtsame Person, die in die Grube gefallen ist « (Fazzioli 1991: 104). Eine mögliche Bedeutung des imaginären Zeichens als Ganzes wäre dann >doppeltes Unglück<. Doch auch dieses Zeichen gibt es realiter nicht.

Bei allen hier vorgestellten Beispielen handelt es sich um einfache Zusammensetzungen. Für die komplexeren Zeichen, wie sie im Buch des Himmels weit häufiger zu finden sind, kombinierte Xu Bing nicht nur Radikale, sondern er modifizierte sie auch, so z.B. durch Hinzufügung einiger Striche oder durch deren Eliminierung. Wir haben es hier demnach mit künstlerischen Zeichenexperimenten zu tun, die ihrerseits jedoch auf höchst rationalen Verfahren beruhen und deren Ziel darin besteht, den Zeichen ihre Bedeutung vollständig zu entziehen, um gleichsam nur Zeichenreste zurückzulassen, die die »Abwesenheit eines Werkes« (Foucault 2003: 175) markieren.

\section{Die Reine Spur der Schrift}

Auf Xu Bings fingierte Zeichen trifft im gewissen Sinne aber auch zu, was Derrida in seinem Aufsatz Die différance über den grafischen Unterschied zwischen Différence und Différance bemerkte: »[...] er läßt sich schreiben oder lesen, aber er läßt sich nicht vernehmen«, d.h. hören (Derrida 1988: 30). Das stumme Spiel der Differenzen (welche er in erster Linie als Schriftspuren begreift) sieht Derrida als die Bedingung der Möglichkeit des Funktionierens eines jeden Zeichens an. Dieser Gedanke ließe sich problemlos auf Xu Bings Zeicheninstallation übertragen, sofern im Zwischenbereich zwischen Zeichen und Nicht-Zeichen Anordnungen von Strichzügen kreiert, modifiziert und unentwegt >differiert< werden, die als ein System leerer Signifikanten erscheinen. Seine Zeichensimulakren laden zur Deutung und Interpretation ein, die sie im selben Moment sabotieren, und inszenieren auf diese Weise den Entzug des Sinns im sprachlichen Sein. Sie eröffnen ein freies, assoziatives Spiel von Differenzen, die der realen, funktionierenden Schrift vorausgehen und im Sinne einer Dekonstruktion der chinesischen Schrift aufgefasst werden könnten (vgl. Köppel-Yang 
2003: 162f.). Mit Derrida ließe sich sagen, dass Xu Bings Bücher des Himmels eine Schrift präsentieren, die so weit modifiziert worden ist, dass sie sich dem Nicht-Sinn öffnet: eine Schrift, für die es keine Lautung gibt und die daher nur grafisch existiert, oder vielmehr: Spuren der Schrift vor jeder Bedeutung.

Mit der Vorstellung einer >Ur-Schrift< hatte Derrida in seiner Grammatologie versucht, sich dem Phonozentrismus der abendländischen Schriftkritik zu entziehen (vgl. Derrida 1974: 99). Er kennzeichnet die Auffassung von Leibniz, der in der chinesischen Sprache das Modell einer philosophischen Sprache erblickte, zwar als ein »Verkennen « und hält sie für gleichsam »rationalistisch und berechnend « (ebd.: 142). Aber er folgt trotzdem in gewisser Weise dem Weg jener Philosophen, die in der chinesischen Schrift eine Alternative zur abendländischen Metaphysik sahen. Derrida bezieht sich in seiner Argumentation (allerdings recht unkritisch) auf den Sprachwissenschaftler Ernest Fenollosa, der bereits Ezra Pound zu einer ideogrammatischen Poetik inspiriert hatte. Dennoch geht er nicht so weit, wie einige seiner Kritiker behaupten, ${ }^{6}$ die chinesische Schrift tatsächlich als >Ur-Schrift< anzusehen, die er in der Grammatologie als ein ursprüngliches Auf- und Verschieben der Präsenz und als eine vorgängige Spaltung jeden Ursprungs beschrieben hat.

$\mathrm{Xu}$ Bings Zeichensimulakren sind im Vergleich zu den realen chinesischen Schriftzeichen in Hinblick auf eine grammatologische Schrifttheorie weit verführerischer. Denn mit ihren unablässigen Abwandlungen markieren sie das »Ende des Buches « und den »Anfang der Schrift« (ebd.: 16), indem sie unter der Voraussetzung eines abwesenden (transzendentalen) Signifikats das entgrenzte Spiel der Differenzen anschaulich machen. Mit anderen Worten: Nicht die chinesische Schrift, sondern Xu Bings Schriftsimulation folgt dem Modell des >freien Spiels< von Zeichenspuren, die sich aus rein räumlichen Differenzen einzelner Striche (Markierungen) zusammensetzen und als leere Signifikanten erweisen. Derrida hätte sie wahrscheinlich als eine Art désécriture bezeichnet, als eine »Dekonstruktion aller Bedeutungen, deren Ursprung in der Bedeutung des Logos liegt« (ebd.: 23).

Begreift man Xu Bings >Kunstschrift< als Inszenierung einer >reinen Spur der Schrift<, dann müsste sie, weil die Spur der Schrift auch eine »Spur vor dem Seienden« (ebd.: 82) ist, notwendig verborgen sein und als Verbergung ihrer selbst entstehen. Das aber würde bedeuten, dass sie sich nur im Verborgenen präsentieren kann, außerhalb der gewöhnlichen und gewohnten Signifikation. Sie kann daher nur in der Exteriorität, d.h. im nicht-signifikativen Niemandsland der Kunst erscheinen.

Im Jahre 2000 kam es zu einer persönlichen Begegnung zwischen Derrida und $\mathrm{Xu}$ Bing, auf die Letzterer sehr lakonisch, ja geradezu unwirsch reagierte: Derridas Bücher seien zu schwierig für ihn, und wenn er sie verstehen würde, dann könne er womöglich seine Kunst nicht mehr fortsetzen.7

6 | Vgl. z.B. Palumbo-Liu 1993.

7 | Vgl. z.B. ebd. 


\section{LITERATUR}

Blanchot, Maurice (1993): Die Literatur und das Recht auf den Tod. In: Ders.: Von Kafka zu Kafka. Frankfurt a.M., S. 11-53.

Derrida, Jacques (1974): Grammatologie. Frankfurt a.M.

Ders. (1988): Die différance. In: Ders.: Randgänge der Philosophie. Wien, S. 29-52.

Eagleton, Terry (1988): Einführung in die Literaturtheorie. Stuttgart.

Fazzioli, Edoardo (1991): Gemalte Wörter. 214 chinesische Schriftzeichen - Vom Bild zum Begriff. Ein Schlüssel zum Verständnis Chinas, seiner Menschen und seiner Kultur. 5. Aufl. Wiesbaden.

Foucault, Michel (1971): Die Ordnung der Dinge. Eine Archäologie der Humanwissenschaften. Frankfurt a.M.

Ders. (2003): Schriften zur Literatur. Frankfurt a.M.

Greenblatt, Stephen (1995): Resonanz und Staunen. In: Ders.: Schmutzige Riten. Betrachtungen zwischen Weltbildern. Frankfurt a.M., S. 7-29.

Harrist jr., Robert E. (2006): Book from the Sky at Princeton: Reflections on Scale, Sense, and Sound. In: Jerome Silbergeld/Dora C.Y. Ching (Hg.): Persistence/Transformation. Text as Image in the Art of Xu Bing. Princeton, S. 25-45.

Köppel-Yang, Martina (2003): Semiotic warfare: A semiotic analysis, the Chinese avant-garde, 1979-1989. Hongkong.

Lloyd, Ann Wilson (2004): Die verschwindende Tusche. In: "Sprachräume". Xu Bing in Berlin. Ausstellungskatalog, Museum für Ostasiatische Kunst. Berlin, S. 22-29.

Middleton, Christopher (1957): Notizen eines Walser-Übersetzers. In: Du. Schweizerische Monatsschrift, H. 10 (Oktober), S. 42-50.

Palumbo-Liu, David (1993): Schrift und kulturelles Potential in China. In: Hans Ulrich Gumbrecht/Ludwig K. Pfeiffer (Hg.): Schrift. München, S. 159-167.

Taylor, Insup/Taylor, M. Martin (1983): The Psychology of Reading. New York/London. Walser, Robert (2003): Aus dem Bleistiftgebiet. Mikrogramme aus den Jahren 1924 bis 1933. 6 Bde. Hg. v. Bernhard Echte u. Werner Morlang. Frankfurt a.M.

Weekes, Brendan/Chen, May Jane/Yin, Bo-lin: Repetition Priming Effects of Chinese Characters and Pseudocharacters. In: Hsuan-Chih Chen (Hg.): Cognitive Processing of Chinese and Related Asian Languages. Hongkong, S. 171-186. 